PAPER

\title{
A polydimethylsiloxane-based axicon lens for focused ultrasonic brain stimulation techniques
}

\author{
Fabián Acquaticci ${ }^{1,2, *}$, Juan F. Guarracino ${ }^{1}$, Sergio N. Gwirc ${ }^{2}$ and Sergio E. Lew ${ }^{1, \dagger}$ \\ ${ }^{1}$ Instituto de Ingeniería Biomédica, Universidad de Buenos Aires, Buenos Aires, Argentina \\ ${ }^{2}$ Instituto Nacional de Tecnología Industrial, Ministerio de Producción, \\ Buenos Aires, Argentina
}

(Received 24 July 2018, Accepted for publication 28 November 2018)

\begin{abstract}
In this work, we built ultrasonic disc-shaped transducer for targeted neuromodulation with the addition of a solid axicon lens based on a polydimethylsiloxane (PDMS) interface. We made a numerical and experimental characterization of its acoustic field. The motor cortex of CF-1 mice was stimulated, through the skin and skull into the intact brain, with low-intensity pulsed ultrasound. Evoked muscle responses in different body segments were clearly observed, including hindlimb, forelimb, and tail. Axicon lens affixed on the face of the transducer makes possible a targeted modulation of the motor cortex by pulsed ultrasound, inducing muscle contraction in a specific body segment. In this approach, the lateral and axial spatial resolution is comparable to spherical segment ultrasound transducers, but with a shorter focal length. Thus, ultrasound axicon looks attractive to investigate the functional contributions of fine-grained spatial structures in the brain.
\end{abstract}

Keywords: Ultrasound axicon, Axicon lens, Focused ultrasound, Neuromodulation, Transcranial stimulation

PACS number: 43.80.Jz, 43.58.Ls [doi:10.1250/ast.40.116]

\section{INTRODUCTION}

The development of a noninvasive, anatomicallytargeted method for controlled modulation of regional brain activity would offers a new opportunity in creating a wide range of applications, from functional brain mapping to the treatment of neurological and neuropsychiatric disorders [1]. Although invasive techniques are finding increasing acceptance in neuro-therapeutics, these methods are accompanied by inevitable risks. As a noninvasive alternative, for example, transcranial magnetic stimulation (TMS) has also been used to modulate cortical activity [2]. Noninvasive methods, however, have limited spatial specificity and depth of penetration, impeding their broad application in brain research and clinical neuroscience [3].

Historically used for medical imaging, ultrasound (US) have recently been shown to be capable of noninvasively stimulating brain activity [4]. These studies were based on the non-targeted application of unfocused acoustic beams to various neural structures. Using low-frequency and lowintensity US stimulation in the intact mouse, [4] reported

*e-mail: facquaticci@fi.uba.ar

†e-mail: slew@fi.uba.ar compelling evidence of transcranial US activation in-vivo, and how pulse application of low-intensity US to regional brain tissue induces changes in the state of neuronal excitability.

One problem associated with an unfocused transducer, built from a single element of diameter $D$ and a $1 / 4$ wavelength matched layer, is its poor lateral resolution. The beam profile (without a focusing method) presents the maximum sound pressure at the near-field distance $(N)$, the natural focus of the system, and has a diameter of $D / 2$. Within the near field, there are maximum and minimum pressure zones in which rapid fluctuations can exits both along and across the sound beam axis [5]. In this sense, the focusing effect of an axicon lens greatly improves the lateral resolution of the transducer, and the near-field zone disappears within the lens [6]. The elimination of the near field, by means of an appropriate axicon lens, enables transducers featuring the same wavelength/diameter ratio $(\lambda / D)$ to produce the same focal spot size. Thus large diameter, low-frequency transducers may be used. This is useful for brain stimulation where low frequencies are required for penetration of the skull. Figure 1 illustrates the natural focus of a transducer at one near field distance and the focusing behavior of an axicon lens. In addition, 

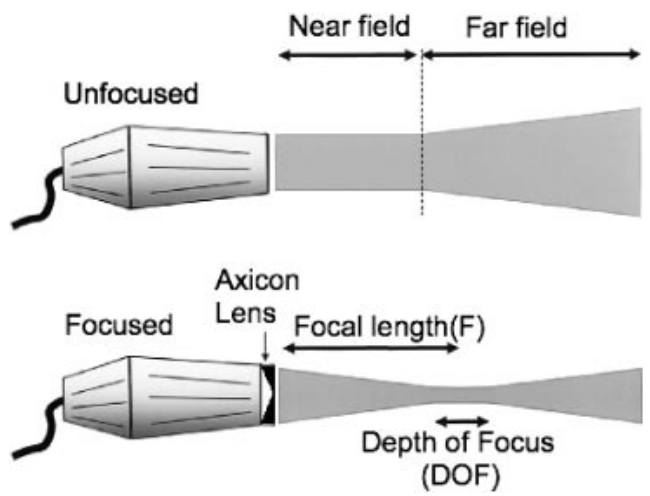

Fig. 1 Illustrative comparison of the focus of a conventional transducer at one near field distance and focusing behavior of an axicon lens contact longitudinal transducer.

depending on the constructive parameters and the materials used for the lens, we can choose different focal lengths and beam diameters.

As a preliminary experiment to study in-vivo feasibility of using axicon lenses to generate transcranial focused ultrasound (tFUS) and transiently modulate region-specific brain activity in mice, we fabricated a prototype of tFUSaxicon transducer and its performance was demonstrated by hydrophone measurements and stimulation tested on the motor cortex. The device was directly coupled to the animal skin to transmit transcranial pulsed US into the brain. The axicon word describes a conical lens formed by a plane and a conical surface. With an axicon lens formed by PDMS, the cavity where the lens is formed is not filled with a liquid, for example oil, so its structure is more reliable. To design a solid and stable axicon lens without degassed liquids in the transducer-lens interface, that can regasify or leak, acoustic field simulations were conducted to obtain proper design specifications for the axicon lens and predict its performance. For this, we have developed a numerical model that takes into consideration the acoustic impedance of the materials used for the lens. We find that the PDMS provides a smaller depth of focus $(D O F)$, which is desirable for neuro-stimulation since it allows a higher axial resolution, comparable to segmented-sphere focused transducers [7-9] but with a shorter focal length, which facilitates the coupling with the head for cortical stimulation. This results in a better and more compact system since the focal spot can be located a few millimeters deep from the face of the focused ultrasound (FUS) transducer.

\section{MATERIALS AND METHODS}

\subsection{Animal Handling}

All experiments were performed according to protocols approved by the University of Buenos Aires Institutional Animal Care and Use Committee, and every precaution was taken in order to minimize stress in animals. To conduct tFUS stimulation of intact motor cortex, CF-1 mice $(n=3)$ were anesthetized with ketamine $(80 \mathrm{mg} / \mathrm{kg}$ i.p.)/Xylazine $(10 \mathrm{mg} / \mathrm{kg}$ i.p.). The proposed anesthetic cocktail provided adequate analgesia, suppressed the spontaneous movement and did not interfere with the evoked motor responses. Ophthalmic ointment was applied to keep the eyes moist. We had previously used a small scissor and depilatory cream on the head of the mice under lighter anesthesia, to remove hair from the scalp, where the ultrasound transducer would be placed.

Once the appropriate level of anesthesia was reached (10 min.), the animal was placed in prone position on a custom-designed holder to secure the mouse's head so that the limbs and the tail were not restricted. Normally there are three points of contact between the device and the mouse, the nose and both ears. Usually there are two spikes on either side of the head that are inserted into the ears. This can damage the mouse's eardrums if not done carefully. We decided to work around the problem by flattening the ends of the spikes and holding the rodents by the back of their jaws. The US-induced cortical stimulation devices were positioned and fixed over the head of the mouse, using an adjustable positioning arm, above the targeted brain region and coupled to the skin using ultrasound gel placed on top of the scalp and on the face of the device while minimizing the introduction of air bubbles, due to the fact that they could interfere with the transmission of US. Figure 2 shows the animal preparation for sonication.

In terms of safety, the acoustic intensity used was below the maximum limits for ultrasound imaging in the FDA guidelines [10], and was conformed with the normal behavior we observed in all animals after brain sonication.

\subsection{Generation of Pulsed US Waveform and In-vivo tFUS Stimulation}

A prototype tFUS-axicon transducer operating at a frequency of $445 \mathrm{kHz}$ was fabricated. The relatively low frequency, compared to frequencies used in diagnostic ultrasound imaging, is applicable to the transcranial delivery of FUS whereby the frequency range of 440$700 \mathrm{kHz}$ has an optimal transmission gain through the $e x$ vivo human skull [11]. The transducer, with the axicon lens, had a diameter $(D)$ of $28 \mathrm{~mm}$, focal diameter $\left(d_{\mathrm{F}}\right)$ of $2.5 \mathrm{~mm}$ and focal length $(F)$ of $10 \mathrm{~mm}$. Figure 3 shows the basic ultrasonic brain stimulation rig for generating the pulsed tFUS. The transducer was actuated by a sinusoidal electrical signal generated from a two-channel function arbitrary waveform generator (Siglent SDG1005). Channel $1(\mathrm{CH} 1)$ was used to establish the acoustic frequency (Af) and the number of cycles per pulse (CpP). Channel 2 $(\mathrm{CH} 2)$ was used to trigger ultrasonic pulses, establish the 


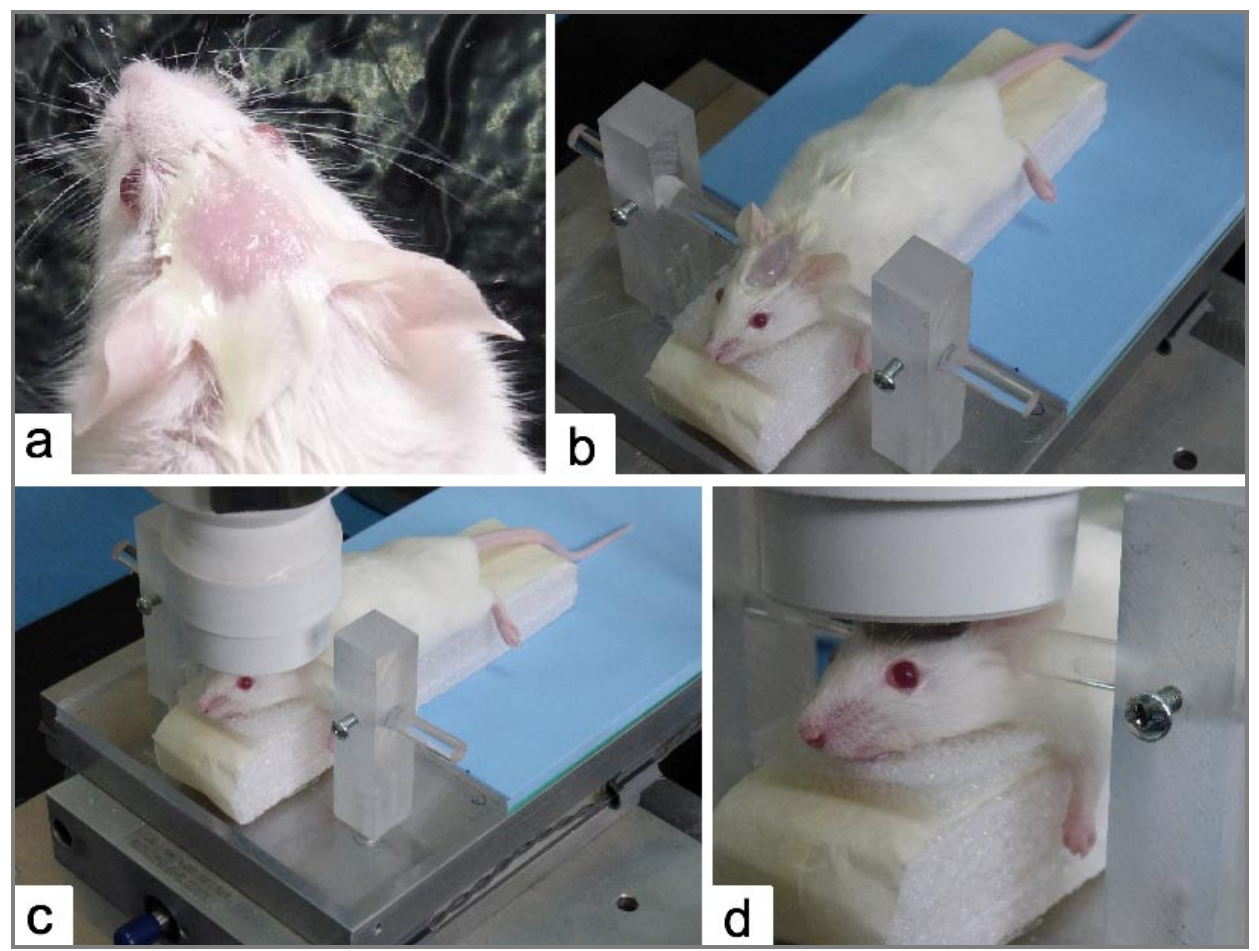

Fig. 2 Preparation of ultrasound-evoked stimulation of intact motor cortex. (a) Mouse with its hair removed from the scalp is shown. (b) Ultrasound gel is shown being placed on top of the scalp of the mouse into a stereotactic positioner. (c, d) The animal's head is coupled to ultrasound transducer by a coupling column containing degassed water.

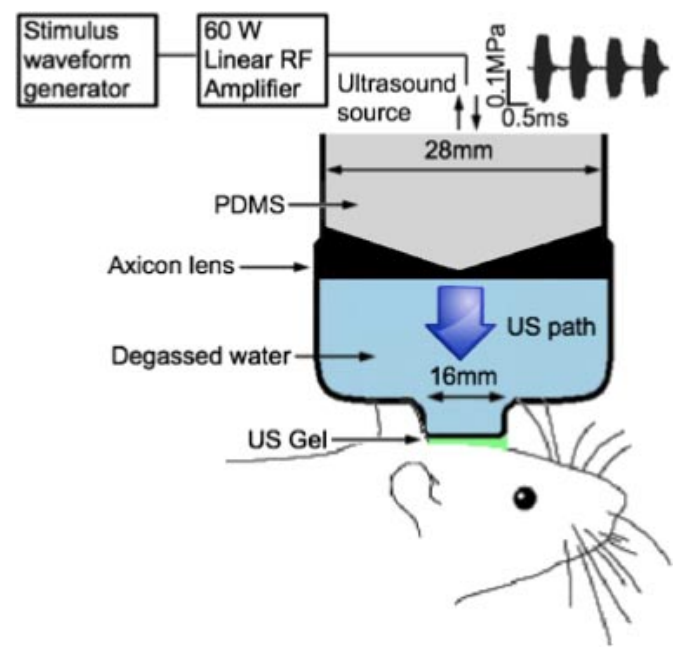

Fig. 3 Experimental setup. The end of the coupling column was sealed with $0.05 \mathrm{~mm}$ polyethylene and sat approximately $2 \mathrm{~mm}$ from the animal's head and between the eye and ear canal.

pulse-repetition frequency (PRF) and define the number of pulses (NP) in a stimulus waveform, which was amplified by a linear RF amplifier (RF Source PM60F2) to provide final voltages to US transducer in order to produce the acoustic pressure profile. The electrical impedance of the transducer was matched to the output impedance of the amplifier.

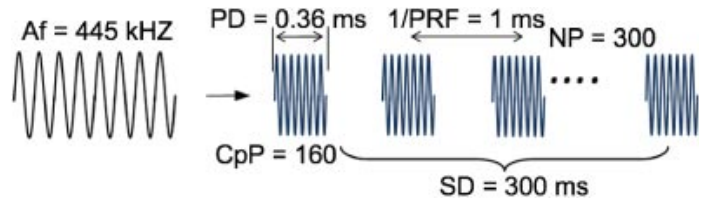

Fig. 4 The sonication parameters used in the experiments.

Figure 4 illustrates the strategy for designing pulsed US stimulus waveforms. Ultrasound can produce thermal and/ or non-thermal (mechanical) bioeffects. Only mechanical energy is used, applying the low-intensity ultrasound stimulation as a train of pulses with large inter-pulse intervals to avoid thermal effects, that are not desired for safety reasons [12]. The parameters can be varied to develop different pulsed US stimulus waveforms. Initially, we refer to other works as a starting point for stimulus waveform generation [4].

For sonication parameters, pulse duration (PD) of $0.36 \mathrm{~ms}$ and PRF of $1 \mathrm{kHz}$ were used. These two parameters affect the intensity characteristics of ultrasound and how it triggers neuronal activity safely. Stimulus duration (SD) is related to the number of pulses necessary to induce ion channels operation. Intensity was estimated by measuring radiant force with an ultrasound power meter (Ohmic Instruments UPM-DT-1AV). Although in larger animals the skull is known to present a significant barrier to the 


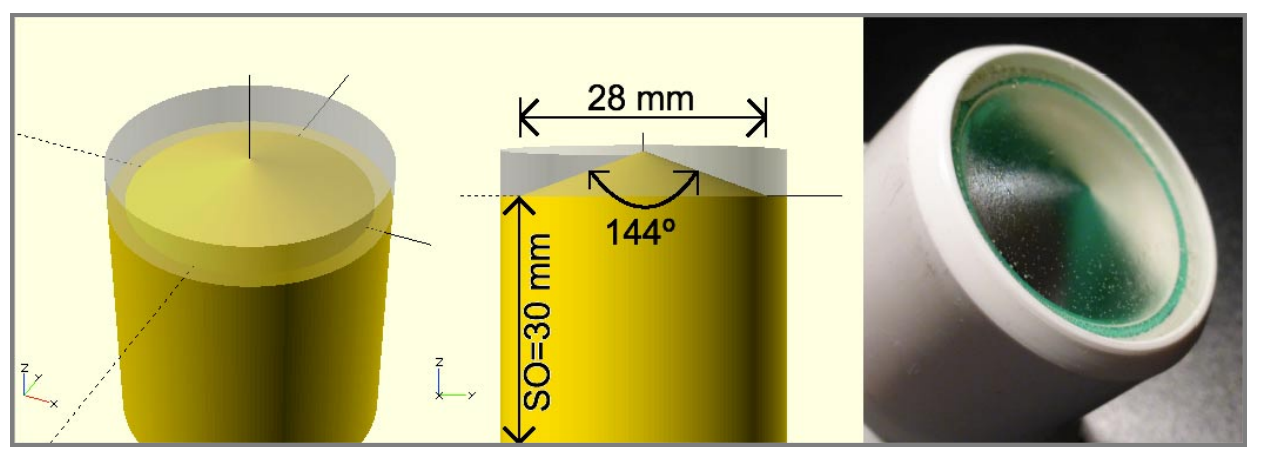

Fig. 5 (Left) Schematic diagram of the axicon lens with its dimensions. (Right) Lens construction: Epoxy resin was used in order to built the lens. We used PDMS to fill the conical cavity in the lens-transducer interface.

propagation of ultrasound, the same does not appear to be true in the mouse [13]. Based on this we ignored the effect of the mouse skull in our calculations of US intensity and beam profile for preliminary tests.

\subsection{Functional Mapping of the Mouse Motor Cortex}

The following protocol provides the details needed to visually observe evoked movements by the tFUS stimulation of intact mouse motor cortex circuits in the right hemisphere. At each site, the US intensity was gradually increased until a movement could be detected, indicating the threshold intensity. Then, if no movement was detected with stimulation reaching this motor threshold, it was considered as non-responsive. Like this, we delineate the area related to the control of tail motility.

Sonication began 15 minutes after the administration of anesthesia. The motor cortex of mice was stimulated every $3 \mathrm{~s}$ with a low-intensity train of tFUS pulses having a spatial peak temporal average intensity (Ispta) of 36 $\mathrm{mW} / \mathrm{cm}^{2}$. We centered the output of the axicon lens covering the right hemisphere $(-1.5 \mathrm{~mm}$ to $-4 \mathrm{~mm}$ from Bregma and $0 \mathrm{~mm}$ to $2.5 \mathrm{~mm}$ lateral of the midline) according to the stereotactic coordinates, using mouse brain atlas plates [14].

\section{4. tFUS-axicon Transducer Design}

The axicon lens is conical in shape and is described by its total included cone angle $(\phi)$. A specially designed axicon lens for the tFUS transducer is proposed as shown in Fig. 5. The design is based on the R. V. Murphy's work for acrylic plastic and oil axicon [15]. A guideline for axicon lens design for the same combination of materials is described elsewhere [16]. The cavity is filled with oil and the ultrasonic lens is formed by the liquid/plastic interfaces. The same liquid is also used for the transducer-lens interface.

In this work, plastic/PDMS combination for the axicon lens was used for brain stimulation in the preliminary experiments. To evaluate the performance and obtain design specifications of our axicon lens, beam profile was computed with the open-source k-Wave Matlab ${ }^{\circledR}$ Toolbox [17]. The model design takes into consideration the acoustic impedances and velocities through the PDMS in the piezo-disc-type element-lens interface, the lens material, and the medium. The piezo-disc-type element and the axicon lens have $28 \mathrm{~mm}$ diameter. The included angle $(\phi)$ of the axicon lens was $144^{\circ}$. The optimal separation between the transducer and axicon lens, called Stand-Off (S.O.), was $30 \mathrm{~mm}$. Note that the frequency of the transducer, angle, and diameter of the axicon lens and the S.O. must be chosen, based on the application, for each particular case. In the design, it was verified that

$$
0.1<F / N \leq 0.3
$$

were $F$ is the desired focal length, and $N$ is the transducer near field distance in the tissue, given by

$$
N=\frac{D^{2} f}{4 v}
$$

were $D$ is the transducer diameter, $f$ is the transducer frequency and $v$ is the sound velocity in the tissue.

Values of $F / N$ between 0.1 and 0.3 generally give the most favorable results, eliminating the near acoustic field of the original transducer and creating a very narrow beam. The focusing effect of the lens on the beam is most pronounced at ratios of $F / N<0.3$. Values of $F / N$ less than 0.1 are rarely used because it gives a very near focus. In addition, small values of $F / N$ result in a severe loss of acoustic energy in the lens system, which requires the use of high intensities beams. On the other hand, values of $F / N>0.4$ gives a profile similar to the transducer alone but removing the near-field.

The diameter $\left(d_{\mathrm{F}}\right)$ of the ultrasound beam for a $-3 \mathrm{~dB}$ drop at the focus was determined by numerical simulation, can be calculated as

$$
d_{F}=2.2 \frac{F}{N} \frac{D}{4}
$$


Table 1 Acoustic properties of materials, distilled water, and tissue.

\begin{tabular}{lcccc}
\hline Material & $\begin{array}{l}\text { Velocity } \\
(\mathrm{mm} / \mu \mathrm{s} @ 2 \mathrm{MHz})\end{array}$ & $\begin{array}{l}\text { Density } \\
\left(\mathrm{g} / \mathrm{cm}^{3}\right)\end{array}$ & $\begin{array}{l}\text { Impedance } \\
(\text { MRayl })\end{array}$ & $\begin{array}{l}\text { Loss } \\
(\mathrm{dB} / \mathrm{cm} @ 2 \mathrm{MHz})\end{array}$ \\
\hline Epoxy $\left(25^{\circ} \mathrm{C}\right)$ & 2.53 & 1.17 & 2.96 & 6.8 \\
PDMS $\left(25^{\circ} \mathrm{C}\right)$ & 1.03 & 1.03 & 1.06 & 5.3 \\
Water $\left(20^{\circ} \mathrm{C}\right)$ & 1.48 & 1.00 & 1.48 & 0.08 \\
Brain & 1.56 & 1.03 & 1.61 & - \\
\hline
\end{tabular}

Table 2 Characteristic values of the axicon lens, obtained from the numerical model in water.

\begin{tabular}{ll}
\hline Parameter & Value \\
\hline Transducer frequency $(f)$ & $0.445 \mathrm{MHz}$ \\
Transducer diameter $(D)$ & $28 \mathrm{~mm}$ \\
Transducer near field in the water $(N)$ & $64.75 \mathrm{~mm}(-6 \mathrm{~dB})$ \\
Ratio of $F / N(F / N)$ & 0.16 \\
Axicon lens angle $(\phi)$ & $144^{\circ}$ \\
Focal length $(F)$ & $10.5 \mathrm{~mm}$ \\
Depth of focus $(D O F)$ & $15.75 \mathrm{~mm}(-3 \mathrm{~dB}) / 26 \mathrm{~mm}(-6 \mathrm{~dB})$ \\
Focus diameter $\left(d_{\mathrm{F}}\right)$ & $2.5 \mathrm{~mm}(-3 \mathrm{~dB}) / 3.5 \mathrm{~mm}(-6 \mathrm{~dB})$ \\
Stand-Off $(S . O)$. & $30 \mathrm{~mm}$ \\
\hline
\end{tabular}

The approximate depth of focus $(D O F)$ of the ultrasound beam for the same drop is given by

$$
D O F=0.38 F \text { to } 1.88 F
$$

Equations (3) and (4), based on Murphy's study, were found experimentally valid for the lens described here. As the ratio $F / N$ decreases, a proportional decrease occurs in $d_{\mathrm{F}}$, and $D O F$ is reduced.

To obtain reliable simulations it is very important to accurately know the propagation velocity of ultrasound through the materials used for the lens. Acoustic properties of these materials were characterized and are shown in Table 1. The sound speed in these materials has a weak dependence on frequency, less than $1 \%$ measured from $500 \mathrm{kHz}$ to $2 \mathrm{MHz}$. The velocity of sound in epoxy resin and PDMS samples was determined by the time of flight technique, using an ultrasonic echoscope (Digital-Echograph 1090 of Karl Deutsch). This measurement was performed in reflection mode with $2 \mathrm{MHz}$ probe (Karl Deutsch S6WB2.25). Attenuation of ultrasound was determined in reflection at the same frequency.

The values of design parameters are summarized in Table 2. We generate the profiles shown in Fig. 6 by simulation, using the aforementioned configuration, for PDMS and various liquids traditionally used in the transducer-lens interface. It can be seen that both the depth of focus and focal length depends on the sound velocity in the material used for the interface, and their sound velocity are shown in Table 3. The PDMS provides a smaller DOF since the sound pressure in the axial direction decreases with a very steep slope, which helps to improve the axial

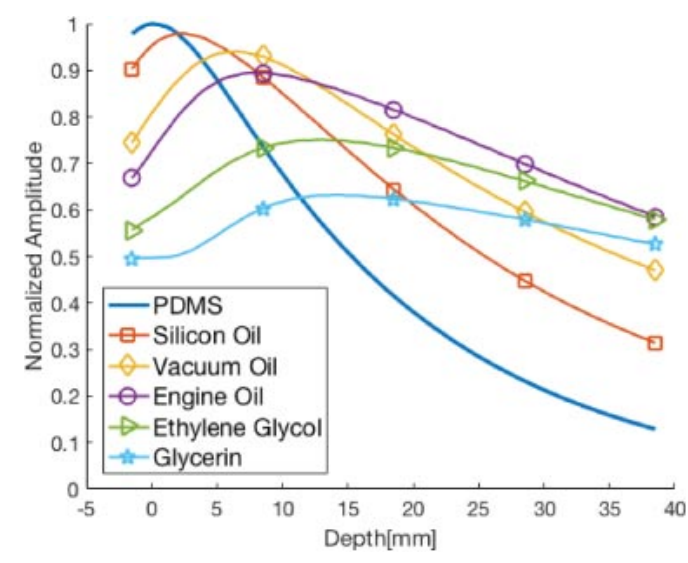

Fig. 6 Simulation of normalized signal amplitude vs. depth of $144^{\circ}$ axicon lenses. We characterized the acoustic field in the axial direction perpendicular to the transducer face (zero depth). The maximum intensity indicates the focal spot.

Table 3 Acoustic velocity of the different liquids and PDMS in the transducer-lens interface. The intention was to cover the largest possible velocity range to obtain a wide diversity of results.

\begin{tabular}{lc}
\hline Medium & $\begin{array}{c}\text { Velocity } \\
(\mathrm{mm} / \mu \mathrm{s})\end{array}$ \\
\hline PDMS (Dow Corning 184) & 1.030 \\
Silicon Oil (Dow Corning 550) & 1.143 \\
Vacuum Oil (Dow Corning 750) & 1.320 \\
Engine Oil (15W40) & 1.478 \\
Ethylene Glycol & 1.685 \\
Glycerin & 1.875 \\
\hline
\end{tabular}


resolution, comparable to spherically focused US transducers. Based on our simulation results, the relation between axicon lens angle $\phi$ and the value of $F / N$ produced is for Epoxy/PDMS lens with Stand-Off:

$$
\phi=\frac{8.8182+\ln \left(\frac{F}{N}\right)}{5.12 \cdot 10^{-2}}
$$

\subsection{Acoustic-field Simulation}

Simulations were based on a k-space pseudo-spectral time domain solution of coupled first-order acoustic equations describing stress and particle velocity within an isotropic 2-D medium. The simulation functions used in kWave require four input structures. These structures define the properties of the computational grid, the material properties of the medium, the properties and locations of any acoustic sources, and the properties and locations of the sensor points used to record the evolution of the pressure field over time [18].

Simulations were performed in two-dimensions. The computational domain was divided into 512 by 512 grid points with a grid point spacing of $250 \mu \mathrm{m}$. The acoustic pressure was recorded at each sensor point for each time step.

To simulate free-field conditions, a perfectly matched layer (PML) is also applied to absorb the waves at the edge of the computational domain [19]. This layer occupies a strip of 20 grid points around the edge of the domain. Without this boundary layer, the computation of the spatial derivates via the FFT causes waves leaving one side of the domain to reappear at the opposite side. The use of PML thus facilitates infinite domain simulations without the need of an increase in the size of the computational grid.

\section{6. tFUS-axicon Transducer Fabrication}

A prototype tFUS-axicon transducer was built following the specifications summarized in Table 2. This transducer has an ultrasonic piezo-disc-type element (Steminc Steiner \& Martins, Inc. SMD28T21F1000R) of PZT-4 mounted on stainless steel housing operating in thickness mode vibration at $445 \mathrm{kHz}$. PZT4 has high Curie temperature and high mechanical quality factor $(Q)$ and, thus, is an excellent material for therapeutic transducer designs. No backing layer was used in the prototype transducer to maximize transmit intensity.

Epoxy (Resoltech 1040) resin was used in order to built the lens with a $\phi$ angle of $144^{\circ}$. In addition, the epoxy used has a high glass transition temperature, which makes it less susceptible to failure during operation. The resin was degassed for 30 minutes into a vacuum chamber and a rubber mold was used to fabricate the conical cavity of the lens inside of a PVC housing. The resin was cured at $40^{\circ} \mathrm{C}$ for $24 \mathrm{hrs}$. After cleaning and drying procedure, the S.O. was filled with degassed PDMS (Dow Corning Sylgard 184). The PDMS mixed solution was cured at $25^{\circ} \mathrm{C}$ for 48 hrs. Figure 7 shows photographs of this fabrication process.

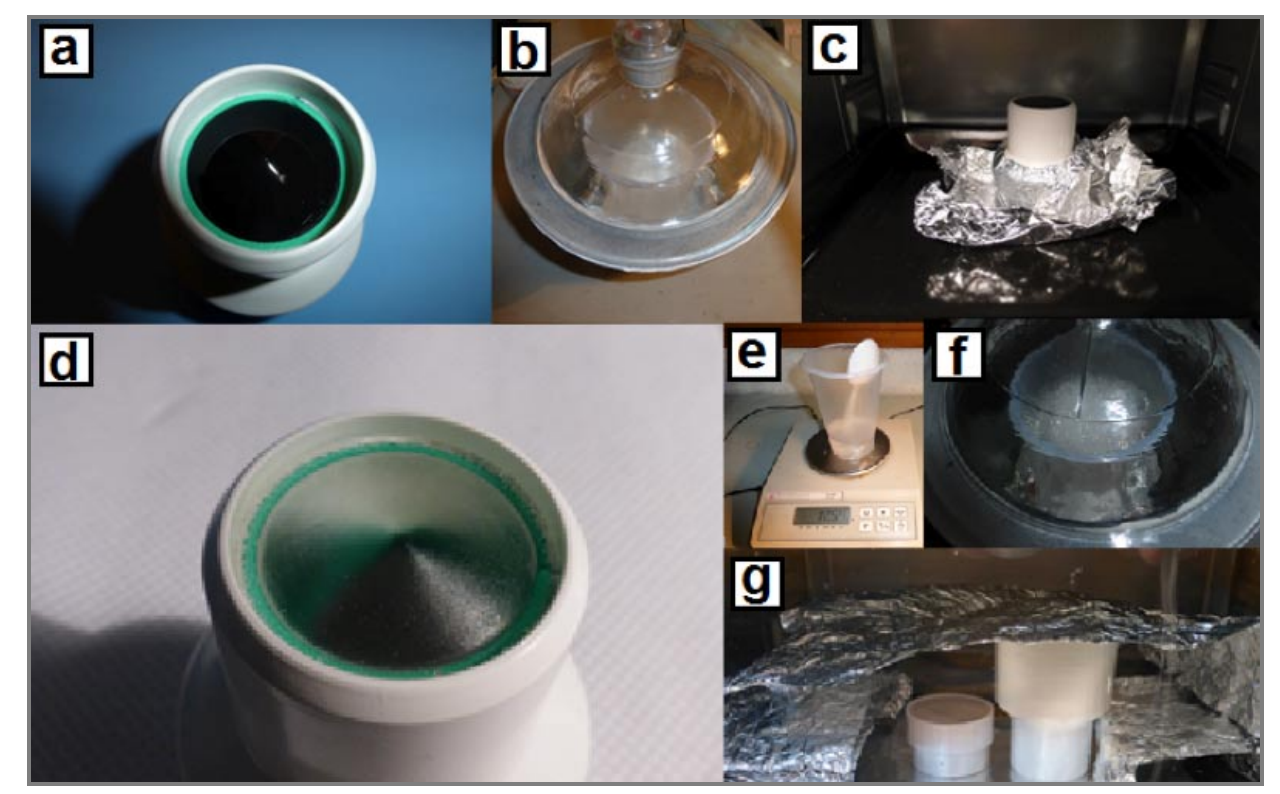

Fig. 7 Fabrication process of Epoxy/PDMS axicon lens. (a) The mold inside of the housing. (b, c) Degassing and curing of the epoxy resin. (d) The conical cavity of the axicon lens. (e) Measurement of a 10:1 ratio of PDMS base to curing agent. (f) Degassing of the PDMS mixed solution into the vacuum chamber. (g) Curing of the PDMS solution inside of the transducer housing. 


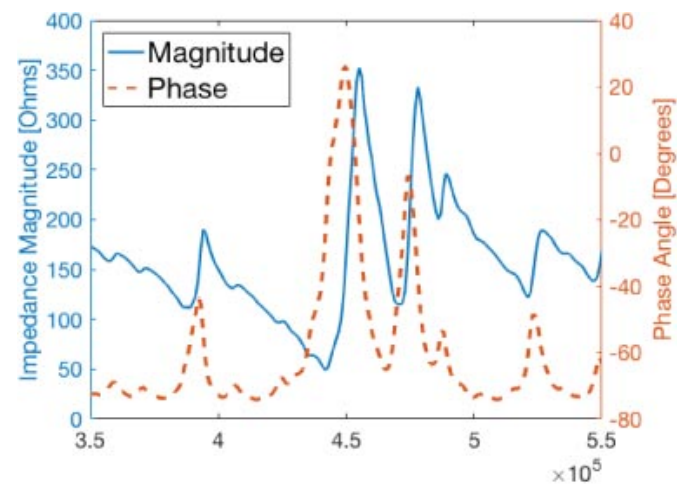

Fig. 8 Measured electrical impedance of the tFUSaxicon after mounted.

\subsection{Electrical Impedance Measurement}

Figure 8 shows the measured electrical impedance of the tFUS-axicon transducer with an impedance analyzer (Agilent 4395A). The transducer element is driven in thickness using a frequency that coincides with a radial harmonic, instead of operating with the main thickness mode of the piezo-disc. The impedance, measured at the operating frequency, was $71 \Omega$ at $445 \mathrm{kHz}$. The transducer electrical impedance should be matched to that of the system, mainly with the power amplifier for balanced output power. The driving frequency was chosen to give a maximal US power value. The principle of measurement is the radiant force method. The transducer was positioned in degassed water above a conical target. The ultrasonic energy passes through the water to reflect off the target and is then absorbed by the rubber lining of the test tank. The radiant power is directly proportional to the total downward force (weight) on the target. This weight is then transferred through a target support assembly to an electromechanical load cell. The cell produces a digital readout in watts of power.

\subsection{Acoustic-field Experimental Measurement}

The objective was to validate our model using experimental measurements of the US field produced by the tFUS-axicon transducer. The experiments were performed in a $230 \times 230 \times 216 \mathrm{~mm}$ test tank, with anechoic coating performed using 3D-printed pyramidal structured absorbers (PSA) [20]. A needle hydrophone (Force Technology MH28) was used to measure the beam pattern of the axicon lens. Both the PSA's and the hydrophone mount have been designed for echo reduction during the measurements as shown in Fig. 9.

The hydrophone scans were performed in degassed water over a rectangular plane of 12,960 grid points through the center of the US field. The swept range for the hydrophone is from $-1.5 \mathrm{~mm}$ to $38.5 \mathrm{~mm}$ in the axial $(y)$ direction, with a step size of $0.5 \mathrm{~mm}$ and from $-19.75 \mathrm{~mm}$ to $20 \mathrm{~mm}$ in the lateral direction $(x)$, with a step of $0.25 \mathrm{~mm}$. The $x-y$ zero point is the focal spot of the axicon lens. The distance between the axicon lens and the hydrophone was varied via a controller (Velmex NF90) with a two-axis positioning system with an accuracy of $\pm 4 \mu \mathrm{m}$ (Velmex MAXY4009).

A function generator with an RF power amplifier (RF Source PM60F2) was used to deliver, through the transducer, a repeated burst of pulsed US at a frequency of

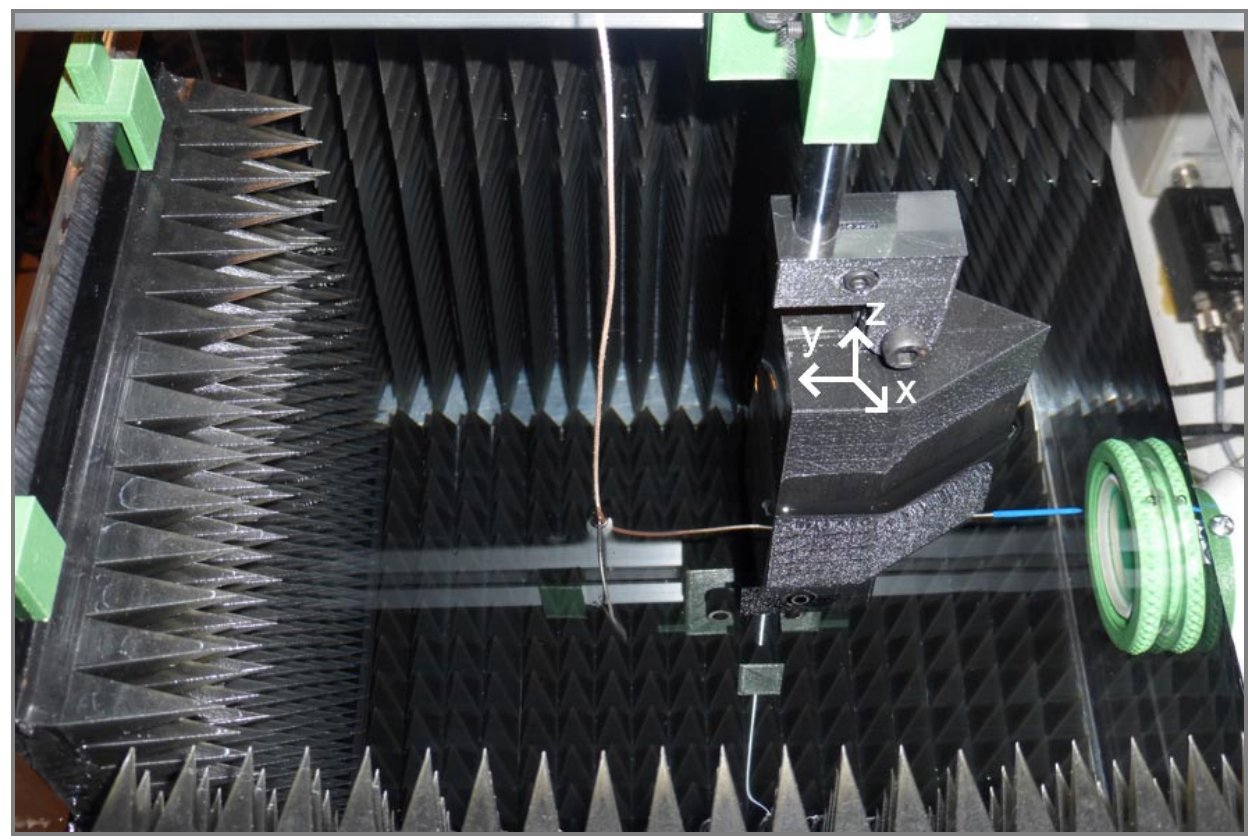

Fig. 9 Photograph of the ultrasound test tank showing the tFUS-axicon transducer, the hydrophone, and the positioning system. 
$445 \mathrm{kHz}$. The signal received by the hydrophone was amplified by $30 \mathrm{~dB}$, measured through an AC millivolt meter (GwInstek GVT-417B) by a true RMS-to-DC converter with 10-bit resolution, and recorded by a computer with data acquisition \& scan control board. The acquisition of RMS value of the pressure at each grid point and subsequent movement of the hydrophone (including a $250 \mathrm{~ms}$ settling time) took just under 2 seconds, with a measurement grid taking on the order of 5 hours to complete.

\section{RESULTS}

\subsection{Targeting tFUS to the Primary Motor Cortex}

Through functional mapping of the mouse motor cortex, we were able to target with tFUS the area related to the control of tail motility of the animals, while no movement was detected in any other body segment. A craniometric approximation was used to locate the target, based on the distance from the eye and ear canal. When using a bilateral-stimulation transducer (without axicon lens), near-simultaneous movements were observed in more than one body segment, including tail, neck, hindlimbs, and forelimbs.

Since we detected no shift in the US focus and no drop in the peak US signal due to the skull, the distribution of neuronal activation it can be correlated in our experiments with the ultrasound field shown in Fig. 10. $Y$-axis refers to the median line of the mouse's skull and $X$-axis, to the bregma. Point zero in the ruler represents the bregma in the

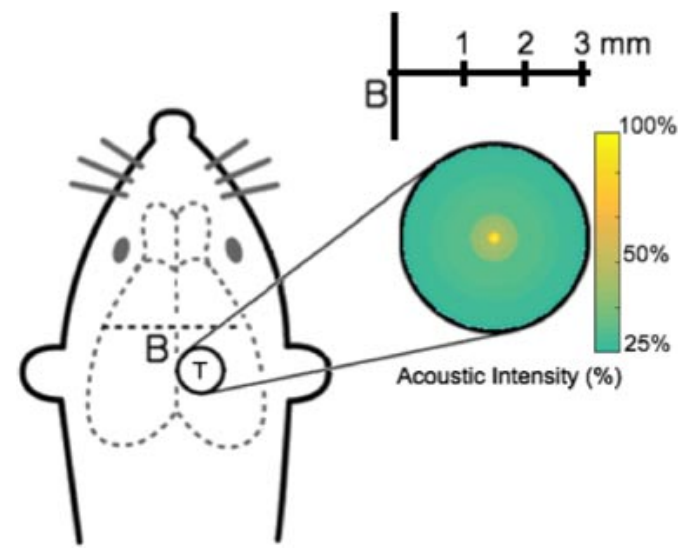

Fig. 10 Top-down view showing the projection of the measured tFUS acoustic field into cortex. Plot illustrates the spatial specificity in the motor cortex with reference to the acoustic intensity drop-off as a function of tFUS beam width. A craniometric approximation was used to locate the target, based on the distance from the eye and ear canal, related to the control of tail motility $(-1.5$ to $-4 \mathrm{~mm}$ from the Bregma and 0 to $2.5 \mathrm{~mm}$ lateral of the midline). By making small adjustments to the positioning of the transducer, the stimulus was delivered to the target area of the motor cortex. antero-posterior axis of the cerebral cortex. The color scale shows the stimulus intensity levels measured in each coordinate in the area related to the control of tail motility. It is an indicative measure of the lateral area of activation offered by US, focusing with acoustic axicon lenses. Small region inside $(d=1 \mathrm{~mm})$ the US brain transmission path is highlighted.

\subsection{Acoustic Beam Properties of tFUS}

In order to assess the accuracy of our simulation results we employed hydrophone scans of the transducer ultrasound field. Simulation using the developed numerical model for the epoxy and PDMS axicon and experimental measurement of both, the cross-sectional profile at the focus and the axial beam profile, are compared in Figs. 11 and 12 , respectively.

The measured and simulated sidelobe level respect to the main lobe was $-11.7 \mathrm{~dB}$ and $-10.3 \mathrm{~dB}$, respectively. The lateral resolution is determined only by the main beam and the level of the lateral lobes is below the neuronal activation threshold by brain stimulation approach using US. The slight deviation between the experimental and simulated results might be due to small alignment errors. In the experiments, the hydrophone was aligned by progressively moving to the center of the beam at varying distances from the lens face. Other sources of errors are uncertainties in the materials properties and the thickness of the thin layer between the conical cavity and the face of the lens. No thermal effects were observed due to weak heating of the PDMS through acoustic absorption.

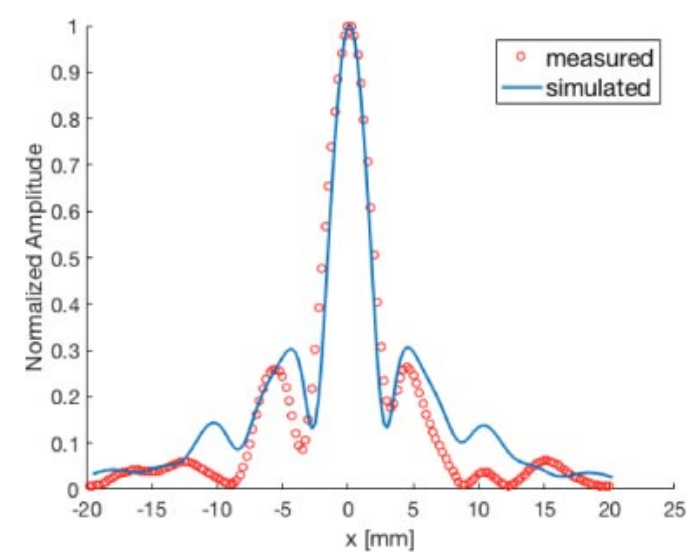

Fig. 11 Comparison of experimental (dotted line) and simulated (solid line) normalized pressure amplitudes of the lateral beam profile produced by the ultrasound axicon. The sidelobes level with respect to the main lobe was $-11.7 \mathrm{~dB}$, with the Stand-Off properly adjusted that improves up to $40 \%$ the lateral spatial resolution, reducing the lateral lobes. The lateral resolution is determined only by the main beam and the level of the lateral lobes is below the neuronal activation threshold by brain stimulation approach using US. 


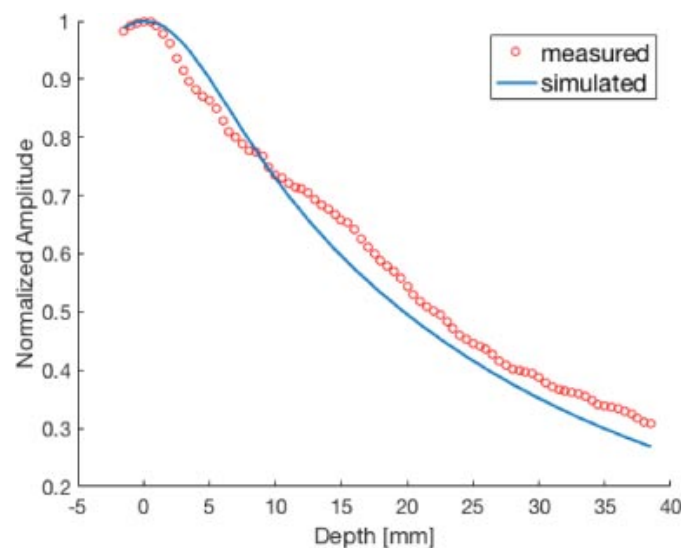

Fig. 12 Comparison of experimental (dotted line) and simulated (solid line) normalized pressure amplitudes of the axial beam profile produced by the ultrasound axicon transducer.

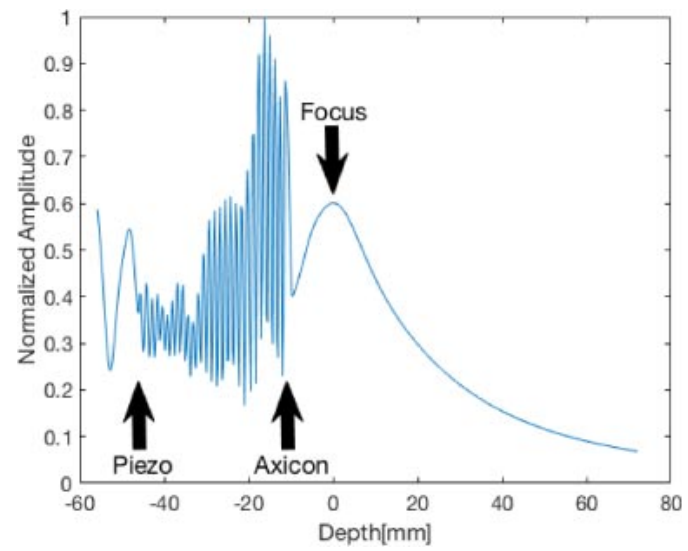

Fig. 13 Simulation of the normalized pressure amplitude vs. depth. The position of the pressure source, the axicon lens, and the focus are indicated. The axicon lens eliminates the near field zone of the standard transducer.

Out of focus, the sound pressure decreases with a very steep slope, as shown in Fig. 13. This sound beam does not exhibit the near-field beam divergence or variations in sound pressure of the transducer without the lens, because the near-field zone remains inside the system that generates the axicon lens. PDMS have better focusing properties for this configuration and geometry of the lens.

Examination of Fig. 14 reveals that there is a good agreement between the simulated and experimental beam patterns. In particular, the diameter $\left(d_{\mathrm{F}}\right)$ of the ultrasound beam at the focus, taken to $\mathrm{a}-3 \mathrm{~dB}$ and $-6 \mathrm{~dB}$ drop, have $2.5 \mathrm{~mm}$ and $3.5 \mathrm{~mm}$ respectively, for both simulated and experimental beam patterns. Figure 14 also shows the hydrophone scan obtained with the same transducer without the use of the axicon lens. In this case, the ultrasound beam at one near field distance has $16 \mathrm{~mm}$ and $19 \mathrm{~mm}$ diameter, for $-3 \mathrm{~dB}$ and $-6 \mathrm{~dB}$ drop of the pressure amplitude respect to the central beam, respectively. This result demonstrates the improvement of the lateral resolution driven by the focusing effect of the axicon lens throughout a depth of focus of $11 \mathrm{~mm}(-3 \mathrm{~dB})$ and $22 \mathrm{~mm}$ $(-6 \mathrm{~dB})$. The insertion loss of the axicon lens at the focus is $8.4 \mathrm{~dB}$, referred to the near field distance $N$ of the transducer without the lens.

\section{DISCUSSION AND CONCLUSION}

There has been long-standing evidence for the efficacy of noninvasive brain stimulation in various neurological disorders: modulation of activity in the targeted cortex (focal epilepsy); modulation of activity in a dysfunctional cortico-subcortical network; restoration of adaptive equilibrium in a disrupted network, guiding plasticity for a best behavioral outcome (stroke); and suppression of plastic changes for functional advantage (pain) [2]. To date, findings are encouraging, but the sham-controlled clinical trial evidence is still insufficient to allow endorsement of the widespread use of tFUS to modulate neuronal activity by delivering anatomically targeted acoustic energy to a localized region in the brain, despite the great margin of safety if appropriate guidelines and precautions are followed. The normal behavior observed in all animals after sonication showed that the tested sonication parameters did not cause brain tissue damage. Our findings support that low-intensity sonication with a small peak pressure amplitude ( $0.1 \mathrm{MPa})$ does not cause the bloodbrain barrier to be disrupted or cavitation. The sonication intensity was far less than the current FDA-regulatory limit on most clinical ultrasound imagers $\left(I_{\text {spta }}=720 \mathrm{~mW} / \mathrm{cm}^{2}\right)$, including obstetrical applications $\left(I_{\text {spta }}=96 \mathrm{~mW} / \mathrm{cm}^{2}\right)$ [21].

There are several methods for delivering ultrasound across the skin and skull, but not all are equally effective. The most accurate yet complicated US focusing method involves the use of multiple transducers operating in a phased array. US can be focused through the skull to targeted brain regions using phased arrays. Focusing with phased arrays is combined with magnetic resonance (MR) imaging to enhance the spatial precision of US localization in a technique known as MR-guided focused ultrasound [4]. The emitted ultrasonic waves will generate a narrow main lobe and low-level side lobes. Nevertheless, this kind of transducers need fairly complex electronic circuitry, have low energy efficiency, low lateral elevation resolution (for linear phased arrays), are large in size and very expensive. On another hand, the most easily implemented method is to use an unfocused transducer for stimulating broad, nonspecific brain regions.

This paper reports the use of axicon lenses to induce neural activation. Their abilities are analyzed in terms of lateral resolution, sidelobe level, depth of focus and focal 

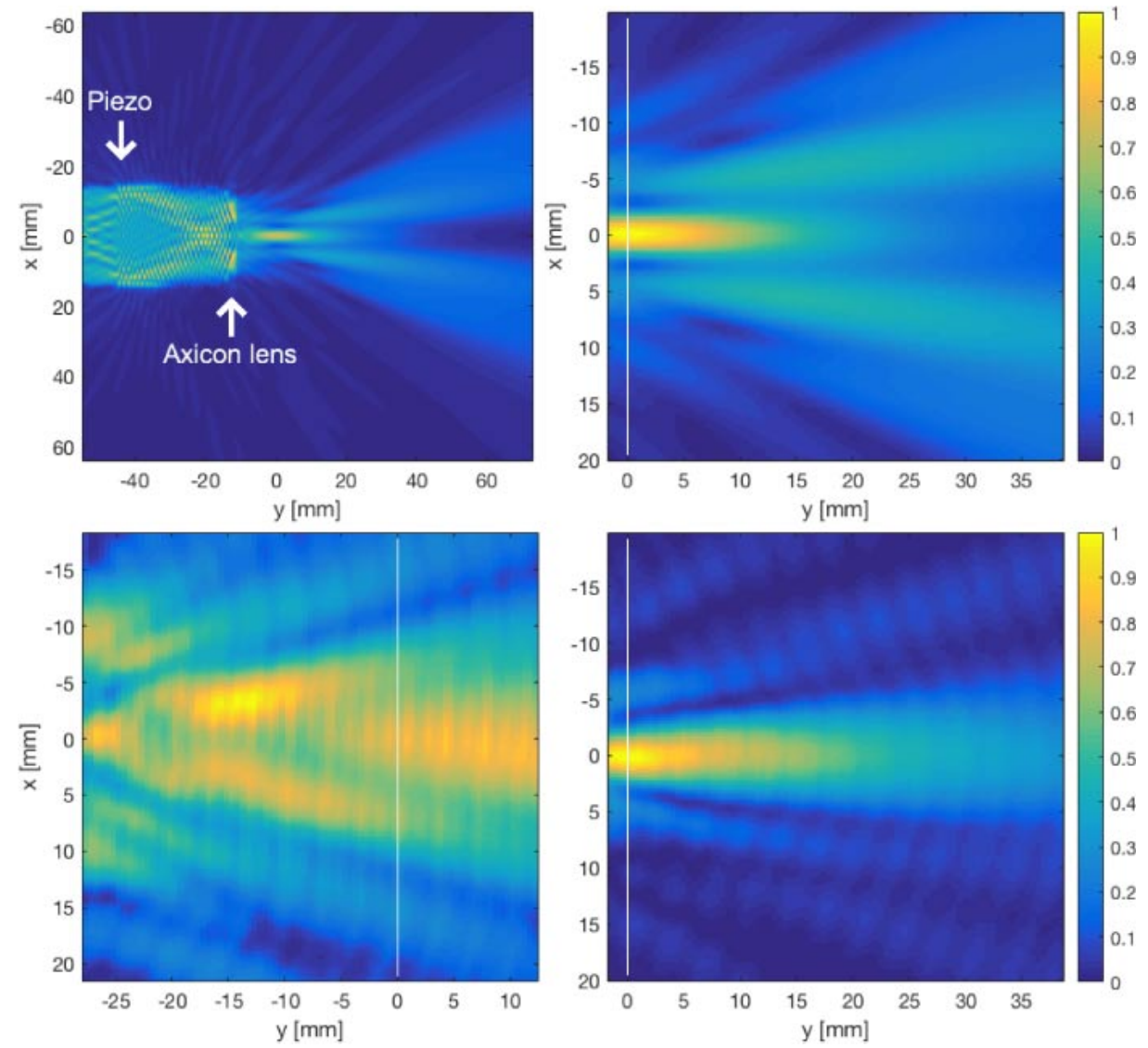

Fig. 14 Normalized acoustic pressure amplitude of the beam patterns in deionized water. (top-left) Simulated acoustic pressure field of the tFUS-axicon transducer. The beam patterns behind the disc-shaped transducer and within the PDMS interface, the axicon lens, and water are shown. The near-field zone disappears within the lens. (top-right) Simulated focusing behavior of the epoxy/PDMS axicon lens. The white line indicate the focus. (bottom-right) Experimental beam pattern produced by the tFUS-axicon transducer with the epoxy/PDMS axicon lens built. The white line indicate the focus. (bottom-left) Experimental beam pattern produced by the ultrasonic piezo-disc-type transducer without the axicon lens. The white line indicate the focus at the near field distance.

spot size, compared to collimated and spherically focused US transducers. With these considerations in mind, it was possible to isolate the movement and delineate the area of the motor cortex related to the control of tail motility of the mice. We show that low-frequency US can be focused using plastic and PDMS axicon lenses having a spatial resolution similar to or better than a spherical segment ultrasound transducer. PDMS turned out to be more reliable than the use of degassed liquids in the transducer-lens interface that can regasify or leak. A good agreement has been found between experimental results and numerical simulations using the k-Wave toolbox.

Axicon lenses have shown to be capable of beam focusing, therefore reducing the beam divergence angle and the focal region (DOF) in comparison with the unfocused transducer's one. In this sense, for brain stimulation, a collimation tubes filled with ultrasound coupling gel, and attached to the same US transducers have been used in others works, but do not eliminate the nearfield and introduce more losses in the transmission path.
Spherically FUS transducer is the most commonly used for focused neuromodulation, but it has a large focal length (several centimeters) that may hinder its coupling with the head. For example, when the focal plane is too close to skull inner ivory layer. The great advantage of the axicon lens is its ability to maintain a very small beam diameter and a very near focus from the lens face outward, and to suppress rapid fluctuations in ultrasound pressure. Nevertheless, if the S.O. is not properly adjusted, internal reflections can occur that prevent this configuration to be used for building focused ultrasound transducers. We are developing a model that takes into consideration the optimal S.O., in order to complete the systematic design of ultrasound axicon lenses for high-resolution brain stimulation.

The ability to induce localized neural activation can ultimately be used as a completely new functional brain mapping method that permits the study of brain connectivity in a much more elegant and noninvasive way than was possible until now. For example, TMS has a limited 
depth of stimulation $(\sim 2-3 \mathrm{~cm})$. Therefore, TMS can readily activate cortical mantle, but cannot reach deeper brain regions (such as hippocampus, amygdala, striatum, thalamus, and brainstem) [22]. Other limitations include the inability to stimulate at high frequencies (over 50 pulses per sec) and the relatively low resolution $(\sim 1-2 \mathrm{~cm})$ [23]. Thus, the TMS cannot be used to investigate the functional contributions from the fine-grained spatial structures such as cortical columns, which contains thousands of neurons possessing similar response specificity. Experimental results with tFUS show that a resolution 10-fold greater than the TMS-associated resolution can be attained. An achievable resolution of $1 \mathrm{~mm}$ might be just enough to resolve cortical columns [24]. The neuromodulatory potential of tFUS can also be used in conjunction with functional neurosurgery, whereby tFUS induces lesions in pathologic neural circuits (for example, epilepsy and movement disorders). The modulatory effect would only be transient, and thus the particular brain regions affected by tFUS sonication can be identified in one session and ablated during subsequent procedures. Other than this imminent potential clinical application, anticipate that the tFUS-mediated modulation of neuronal activity may also open new avenues in clinical applications for the treatment of various neurological and psychiatric illnesses.

Further testing is needed to verify that the human skull is not an obstacle for transcranial focusing of ultrasound by axicon lenses and these do not produce heating of the skin or skull bone.

\section{ACKNOWLEDGMENTS}

The authors would like to thank Dr. Adriana Losavio for allowing to do some of the animal experiments in her laboratory of neurophysiology. We also acknowledge support from IDIM-CONICET and IBYME-CONICET. This work has been partially supported by the Peruilh grant of the University of Buenos Aires, Faculty of Engineering.

\section{REFERENCES}

[1] K. E. Hoy and P. B. Fitzgerald, "Brain stimulation in psychiatry and its effects on cognition," Nat. Rev. Neurol., 6, 267-275 (2010).

[2] F. Fregni and A. Pascual-Leone, "Technology insight: Noninvasive brain stimulation in neurology-perspectives on the therapeutic potential of rTMS and tDCS," Nat. Clin. Pract. Neurol., 3, 383-393 (2007).

[3] T. Wagner, A. Valero-Cabre and A. Pascual-Leone, "Noninvasive human brain stimulation," Annu. Rev. Biomed. Eng., 9, 527-565 (2007).

[4] Y. Tufail, A. Yoshihiro, S. Pati, M. M. Li and W. J. Tyler, "Ultrasonic neuromodulation by brain stimulation with transcranial ultrasound," Nat. Protoc., 6, 1453-1470 (2011).
[5] J. Krautkramer and H. Krautkramer, Ultrasonic Testing of Materials, 2nd ed. (Springer-Verlag, New York, 1977), pp. 6280.

[6] L. M. Soroko, Meso-Optics Foundations and Applications (World Scientific Publishing, Singapore, 1996), pp. 5-12.

[7] S.-S. Yoo, et al., "Transcranial focused ultrasound to the thalamus alters anesthesia time in rats," Neuroreport, 22, 783787 (2011).

[8] W. Lee, et al., "Image-guided transcranial focused ultrasound stimulates human primary somatosensory cortex," Sci. Rep., $\mathbf{5}$, 8743 (2015).

[9] W. Legon, et al., "Transcranial focused ultrasound modulates the activity of primary somatosensory cortex in humans," Nat. Neurosci., 17, 322-329 (2014).

[10] F. A. Duck, "Acoustic saturation and output regulation," Ultrasound Med. Biol., 25, 1009-1018 (1999).

[11] P. J. White, G. T. Clement and K. Hynynen, "Longitudinal and shear mode ultrasound propagation in human skull bone," Ultrasound Med. Biol., 32, 1085-1096 (2006).

[12] W. D. O’Brien Jr., "Ultrasound-biophysics mechanisms," Prog. Biophys. Mol. Biol., 93, 212-255 (2007).

[13] K. Hynynen, et al., "Preclinical testing of a phased array ultrasound system for MRI-guided noninvasive surgery of the brain: A primate study," Eur. J. Radiol., 59, 149-156 (2006).

[14] K. B. J. Franklin and G. Paxino, The Mouse Brain in Stereotaxic Coordinates, 3rd ed. (Academic Press, New York, 2007).

[15] R. V. Murphy, "Focussed ultrasonic probes for contact inspection,” Mater. Eval., September, pp. 53-58 (1980).

[16] P. Katchadjian, C. Desimone and A. D. Garcia, "Application of axicon lenses in ultrasonic techniques," AIP Conf. Proc., 1211, 1043-1050 (2010).

[17] K. Wang, E. Teoh, J. Jaros and B. E. Treeby, "Modeling nonlinear ultrasound propagation in absorbing media using the k-Wave Toolbox: Experimental validation," IEEE Int. Ultrason. Symp., pp. 523-526 (2012).

[18] B. E. Treeby and B. T. Cox, "k-Wave: MATLAB toolbox for the simulation and reconstruction of photoacoustic wavefields," J. Biomed. Opt., 15(2), 021314 (2010).

[19] T. K. Katsibas and C. S. Antonopoulos, "A general form of perfectly matched layers for three-dimensional problems of acoustic scattering in lossless and lossy fluid media," IEEE Trans. Ultrason. Ferroelectr. Freq. Control, 51, 964-972 (2004).

[20] F. Acquaticci, M. M. Yommi, S. N. Gwirc and S. E. Lew, "Rapid prototyping of pyramidal structured absorbers for ultrasound," Open J. Acoust., 7, 83-93 (2017).

[21] AIUM-Committee CS, "How to interpret the ultrasound output display standard for higher acoustic output diagnostic ultrasound devices: Version 2. J.," Ultrasound Med., 23, 723-726 (2004).

[22] M. W. Sliwinska, S. Vitello and J. T. Devlin, "Transcranial magnetic stimulation for investigating causal brain-behavioral relationships and their time course," J. Vis. Exp. (89) (2014).

[23] P. T. Huerta and B. T. Volpe, "Transcranial magnetic stimulation, synaptic plasticity and network oscillations," J. NeuroEng. Rehabil., 6, 7 (2009).

[24] C. Stippich, Ed., Clinical Functional MRI: Presurgical Functional Neuroimaging (Medical Radiology) (Springer-Verlag, Berlin/Heidelberg, 2015), p. 24. 